\title{
Femtosecond Laser Synthesis of Multi-Element Nanocrystals
}

L. N. Dinh, T. Trelenberg, B. Torralva, B. C. Stuart, M. Balooch

\section{January 8, 2003}

U.S. Department of Energy

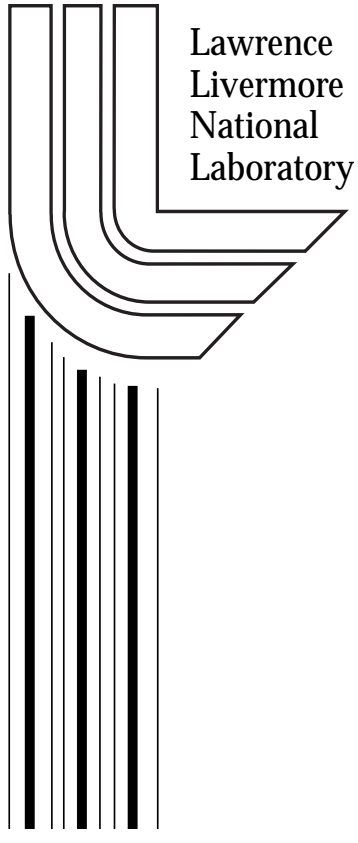




\section{DISCLAIMER}

This document was prepared as an account of work sponsored by an agency of the United States Government. Neither the United States Government nor the University of California nor any of their employees, makes any warranty, express or implied, or assumes any legal liability or responsibility for the accuracy, completeness, or usefulness of any information, apparatus, product, or process disclosed, or represents that its use would not infringe privately owned rights. Reference herein to any specific commercial product, process, or service by trade name, trademark, manufacturer, or otherwise, does not necessarily constitute or imply its endorsement, recommendation, or favoring by the United States Government or the University of California. The views and opinions of authors expressed herein do not necessarily state or reflect those of the United States Government or the University of California, and shall not be used for advertising or product endorsement purposes.

This report has been reproduced directly from the best available copy.

Available electronically at http://www.doc.gov/bridge

Available for a processing fee to U.S. Department of Energy

And its contractors in paper from

U.S. Department of Energy

Office of Scientific and Technical Information

P.O. Box 62

Oak Ridge, TN 37831-0062

Telephone: (865) 576-8401

Facsimile: (865) 576-5728

E-mail: reports@adonis.osti.gov

Available for sale to the public from

U.S. Department of Commerce

National Technical Information Service

5285 Port Royal Road

Springfield, VA 22161

Telephone: (800) 553-6847

Facsimile: (703) 605-6900

E-mail: orders@ntis.fedworld.gov

Online ordering: http://www.ntis.gov/ordering.htm

OR

Lawrence Livermore National Laboratory

Technical Information Department's Digital Library

http://www.llnl.gov/tid/Library.html 


\title{
Femtosecond Laser Synthesis of Multi-Element Nanocrystals
}

\author{
L. N. Dinh, T. Trelenberg, B. Torralva, B. C. Stuart, M. Balooch \\ Lawrence Livermore National Laboratory \\ Livermore, CA
}

\begin{abstract}
We studied the conditions under which short-pulsed laser deposited (PLD) stoichiometric multi-element nanocrystals of GaAs,InP,CoPt and Inconel (an alloy of $\mathrm{Cr}, \mathrm{Fe}$ and $\mathrm{Ni}$ ) are formed. The properties of the PLD nanoclusters and the irradiated targets were investigated as a function of the laser pulse-length (150 fs-500 ps) and the inert background gas pressure in the synthesis chamber (microTorr to hundreds of Torr). Our results reveal that the formation of stoichiometric GaAs nanocrystals required ablating a GaAs target with a shorter than 25 ps laser in a $\geq 50$ miliTorr of inert background pressure. For InP, a mixture of stoichiometric InP and In nanocrystals with an InP/In ratio of $\sim 1$ resulted upon ablating an $\mathrm{InP}$ target in $\mathrm{Ar}$ at 1 Torr. This InP/In ratio increased to $\sim 5$ when ablating the InP target in an Ar pressure of 750 Torr. In case of CoPt alloy, the stoichiometry in the target was not reflected in the collected nanocluster films, independent of the background gas pressure. Interestingly, the stoichiometry of the target was found in the collected nanocluster films when an Inconel target was ablated by a femtosecond laser even in vacuum. It is noted that the constituents of Inconel ( $\mathrm{Cr}, \mathrm{Fe}$ and $\mathrm{Ni}$ ) have similar vapor pressures while $\mathrm{Co}$ and $\mathrm{Pt}$ do not. Our experimental results suggest that the stoichiometries of the PLD multi-element nanoclusters are closer with those of the targets when shorter than $25 \mathrm{ps}$ lasers are used. However, this does not imply that simply irradiating a multi-element target in vacuum with a shorter than 25 ps pulse-length laser would automatically result in the formation of stoichiometric nanocrystals. The preservation of the stoichiometry of the irradiated target and the formation of stoichiometric semiconductor nanocrystals require ablating the targets with a shorter than $25 \mathrm{ps}$ laser in a background gas. The minimum background gas pressure is materials dependent. And for metal alloys, the stoichiometry of the ablated target cannot be found in the collected nanocluster films if the constituent elements have widely different vapor pressures. We have also successfully used density-functional based tight-binding potentials to study the femtosecond laser/GaAs target interaction. This code work can be extended to other multi-element compounds/alloys as well.
\end{abstract}




\section{Introduction:}

Nanoscale materials, particularly three-dimensionally confined structures such as quantum dots or nanocrystals are extremely interesting in that their electronic, magnetic, and physical properties can be tailored to be different than those of bulk materials simply by controlling the size of the nanocrystals. ${ }^{1-3}$ Many novel properties of nanocrystals remain undiscovered due to the difficulty in obtaining high purity nanoparticles, specifically alloy and multi-element nanocrystals with proper stoichiometry. In this report, the properties of the short-pulsed laser deposited (PLD) nanoclusters and the irradiated targets that produced them were investigated as a function of the laser pulselength (150 fs-500 ps) and the inert background gas pressure in the synthesis chamber (microTorr to hundreds of Torr). The conditions under which PLD stoichiometric multielement nanocrystals of compound-semiconductors and metal alloys are formed will be discussed based on the results from ablation of GaAs, InP, CoPt and Inconel (an alloy of $\mathrm{Cr}, \mathrm{Fe}$ and $\mathrm{Ni}$ ). Computational modeling of the short pulsed laser materials interaction was also pursued.

\section{Experiments:}

The experiments were carried out in a vacuum chamber with a base pressure of $10^{-6}$ Torr and with provisions for admitting a buffer gas $\left(\mathrm{SF}_{6}\right.$ or Ar). The ablation laser was a Ti-sapphire laser with tunable pulse length from 500 ps to $150 \mathrm{fs}$, operating at 820 $\mathrm{nm}, 1000 \mathrm{~Hz}$. During ablation, the laser struck the target at a $45^{\circ}$ angle from the target normal and had a fluence of $\sim 0.8 \mathrm{~J} / \mathrm{cm}^{2}$. Rastering and rotation of the ablation target prevented the target from being ablated in the same area repeatedly without allowing for some recovery time. The films were deposited onto Si substrates that were positioned $\sim 11 \mathrm{~cm}$ from the target along the target normal. After removal from the vacuum chamber, the films were transported in air to the different analysis stations designed to perform x-ray diffraction (XRD), Auger electron spectroscopy (AES), x-ray photoelectron spectroscopy (XPS), scanning electron microscopy (SEM), and atomic force microscopy (AFM). Sputtering removal of the outer oxide layers was implemented when necessary.

\section{Experimental Results and discussion:}

\section{A. Compound semiconductors:}

\section{GaAs:}

Fig. 1 shows an AFM image of a PLD GaAs nanocrystal film created in this work. Readers interested in the details of our work on GaAs are referred to our published and submitted papers. ${ }^{4-5}$ However, the main results are summarized below: X-ray diffraction from GaAs films grown in vacuum using different laser pulse lengths (150 fs $-500 \mathrm{ps}$ ) showed diffraction peaks consistent with those expected for GaAs. Crystallite size were $\sim 7 \mathrm{~nm}$ for all but the longest pulse length used ( $\sim 500 \mathrm{ps})$, where both ablation yield and crystallite size dropped dramatically. Producing crystallites of 
only $1.5 \mathrm{~nm}, 500$ ps appears to be near the maximum laser pulse length that will allow the formation of GaAs crystallites in vacuum. AES analysis of the in-vacuum deposited films, however, revealed that the GaAs crystallite observed by XRD had been codeposited with amorphous gallium and arsenic. For all pulse lengths available (150 fs - $500 \mathrm{ps}$ ), films deposited in vacuum were found by AES to contain either excess Ga or As when compared to the virgin GaAs target. It was, however, found that the target stoichiometry was reproduced in the ablated films when the amorphous atomic species were scattered using a background gas of $\geq 50 \mathrm{mTorr}$. The distribution of particles ablated in vacuum as a function of angle was also investigated. The distributions examined at two different pulse lengths both consisted of a tightly forward focused component and a more highly dispersed component. For the highly dispersed component, the order of the fit was the same in both cases but had a far more prominent contribution to the overall intensity in the longer pulse length case, providing evidence that two materials removal mechanisms (a fast non-thermal and a slow thermal processes) occur in the short-pulse laser ablation. When the buffer gas pressure was increased to $\sim 500$ Torr, even the target stroichiometry was also found to be unaffected by the ablation process.

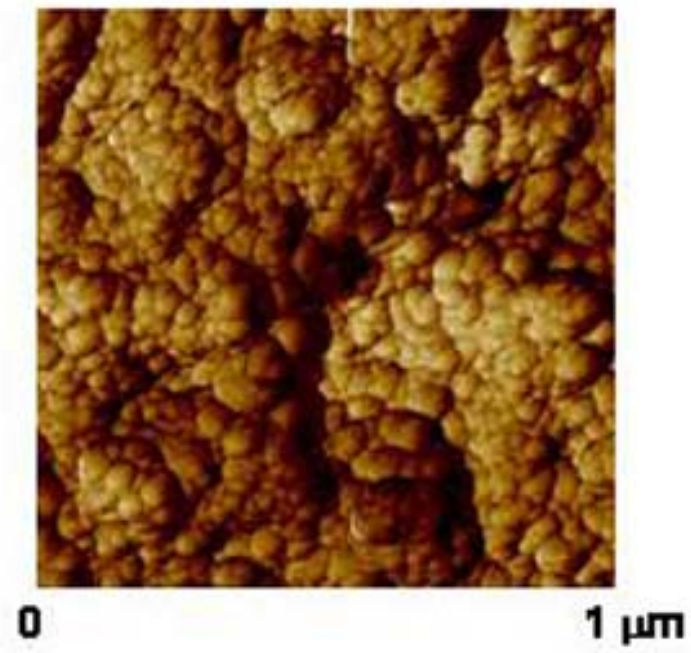

Fig. 1: PLD GaAs nanocrystal film

\section{InP:}

For InP, a mixture of stoichiometric InP and In nanocrystals with an InP/In ratio of $\sim 1$ resulted upon ablating an InP target in Ar at $\sim 1$ Torr. This InP/In ratio increased to $\sim 5$ when ablating the InP target in an Ar pressure of $\sim 750$ Torr. These results are summarized in Fig. 2. Increasing the background pressure during ablation of InP did improve the composition of the films. However, stoichiometric deposition was never achieved even near atmospheric pressure. In GaAs, a background pressure on the order of $100 \mathrm{mT}$ Trr was sufficient to preserve the stoichiometry of the collected films. The success of background scattering for GaAs and its failure with InP can be traced to the masses of the constituent elements relative to those of the compounds. For GaAs, each of the constituent atoms carries roughly half of the mass of the compound. A background pressure which scatters one element will scatter the other. Chemically combined GaAs, having twice the mass of the individual atoms, and bigger GaAs nanoclusters arrive at the 
substrate relatively unimpeded. This is not the case with InP. Indium has an atomic weight that is roughly $371 \%$ that of phosphorus and contains nearly $79 \%$ of the total mass of InP. A background pressure sufficient to scatter the phosphorus will go virtually unnoticed by the atomic indium. Likewise, pressures that begin to reduce the amount of indium reaching the substrate will also serve to scatter the desired InP. Increasing background pressures, therefore, has much less of an effect in increasing the stoichiometry of PLD InP films as in the case of semiconductor compounds with constituents having nearly equal masses such as GaAs.

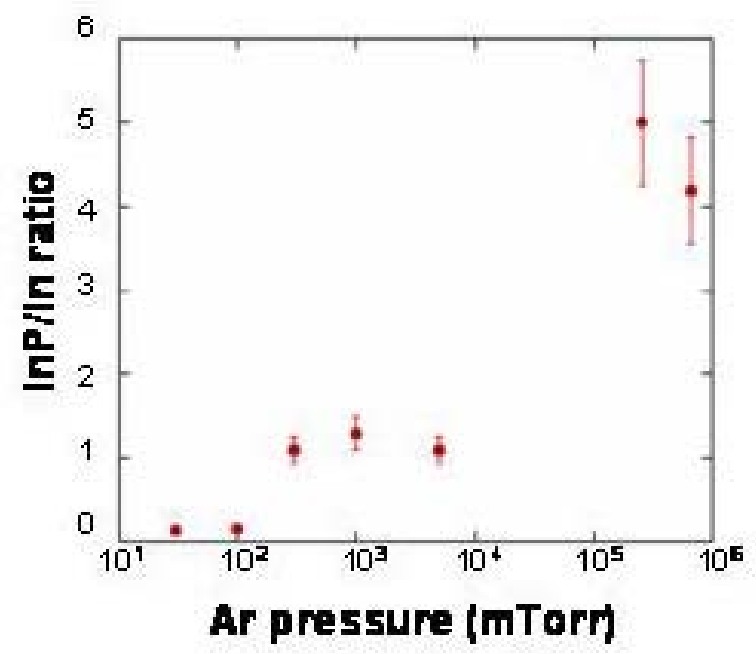

Fig. 2: InP/In ratio in PLD films vs. inert background pressure

\section{B. Metal alloys:}

\section{CoPt:}

Comparison of the XRD spectra from the virgin CoPt target and the laser ablated spots on the same target shows that the original structure and $\mathrm{Co} / \mathrm{Pt}$ ratio were not preserved in the ablation process, with only a slight improvement from the use of very short pulse lengths. ${ }^{6}$ Combined XPS and XRD results indicate that the ablated spots on the CoPt target was rich in Co at the surface (top few nanometers) but deficient in Co just below the top surface. ${ }^{6}$ This observation can be understood as following:

Cobalt, having a much lower melting point and much higher vapor pressures than Pt over the temperature range of interest diffused more readily from the heat-affected zone formed by the ablation process toward the target surface where thermal evaporation from cobalt is also more favorable than Pt. This explains why the ablated spots on the target was found to be rich in Pt (or deficient in Co) by XRD. However, cobalt's lower melting temperature resulted in liquid cobalt on the surface of the target long after the platinum has re-solidified. This explains the high cobalt concentration found by XPS at the top surface layer of the ablated spot.

Even though the addition of a background gas during ablation improves the stoichiometry of compound-semiconductor nanocrystal films as described above, XRD showed that increasing the background gas pressures had virtually no effect in preserving 
the stoichiometry of the CoPt target during ablation or in improving the stoichiometry of the PLD alloy films.

Angular distribution measurements conducted during an in-vacuum ablation, exhibited $\cos ^{35} \theta+\cos \theta$ dispersal of the ejected particles about the target normal. Fig. 3 shows the dual cosine fit to the particle distribution. The $\cos ^{35} \theta$ term is believed to be due to sub-picosecond laser-target interaction processes, while the $\cos \theta$ distribution resembles a thermal source that expels particles effusively. AFM images of the clusters associated with each component of the distribution are shown in the insets of Fig. 3. These images indicate that the forward focused distribution is composed primarily of large clusters (hundreds of nanometers), while much smaller clusters make up the broad cosine distribution.

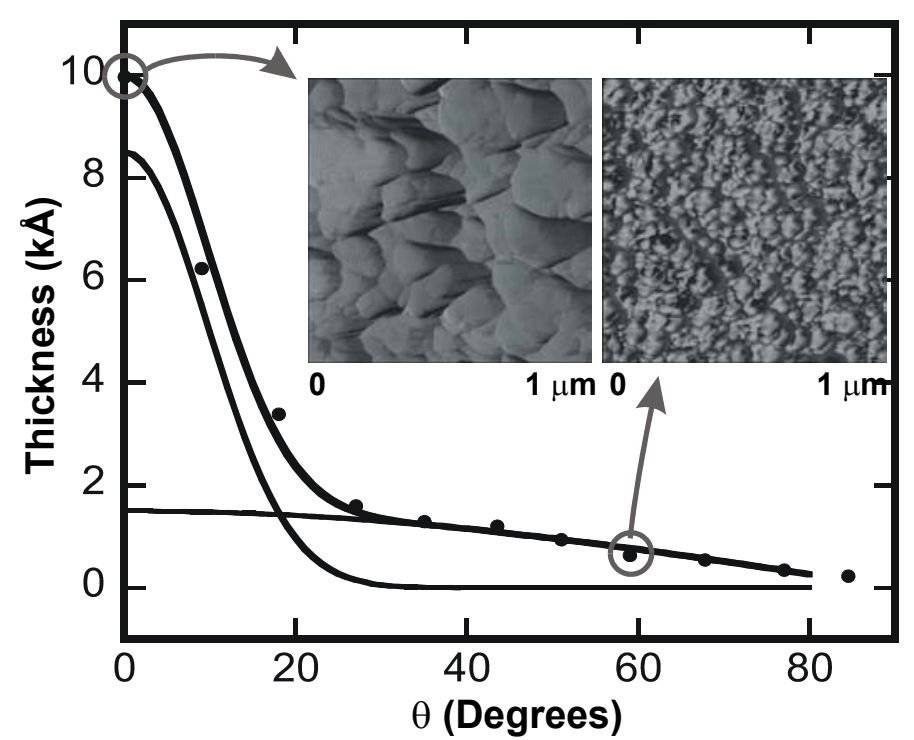

Fig. 3: The dual cosine fit to the particle distribution. AFM images of the clusters associated with each component of the distribution are shown in the insets

\section{Inconel:}

Interestingly, the stoichiometry of the target was found in the collected nanocluster films when an Inconel target was ablated by a femtosecond laser even in vacuum. In Fig. 4, the x-ray fluorescent spectra of a virgin Inconel target (lower) and after laser exposure (upper) show no difference in the target composition. The main difference between the CoPt alloy and the Inconel systems is that the vapor pressures of the constituents in Inconel are much closer to one another over the temperature range of interest than those in the CoPt alloy. 


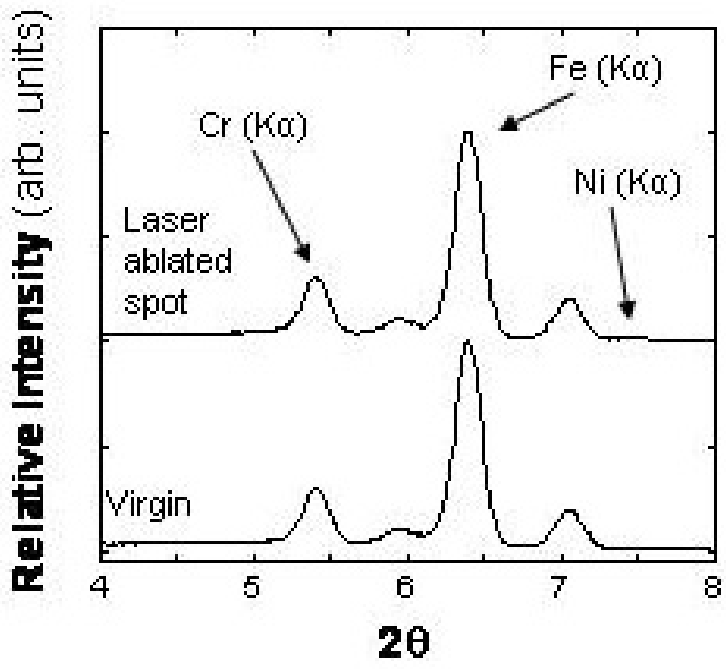

Fig. 4: X-ray fluorescent spectra of a virgin spot (lower) an ablated spot (upper) of an Inconel target

\section{Modeling effort:}

Our modeling effort of the short-pulsed laser compound materials interaction centered on the development of the density-functional based tight-binding electron-ion dynamics model-TED. The effect of short-pulsed laser materials interaction employing TED modeling was first calibrated against silicon prior to applying it on GaAs (110). ${ }^{8}$ This is the main cleavage surface of GaAs and has therefore been extensively studied. The surface does not reconstruct, however it does undergo a relaxation that includes a transfer of charge from the Ga cations to the As anions. The transfer of charge as well as other aspects of the multi-component nature of binary compounds provides additional challenges from a modeling perspective. We have again chosen to use densityfunctional based $\mathrm{sp}^{3}$ tight-binding potentials. To explore the interaction of GaAs with an external laser field we exposed the surface to a 150 fs laser pulse, having a Gaussian-like profile, directed at $45^{\circ}$ with respect to the normal of the surface. The energy of the pulse was centered at $1.5 \mathrm{eV}$, and the fluence was $1.6 \mathrm{KJ} / \mathrm{m}^{2}$. The interaction with the pulse leads to the excitation of 6.9 percent of the electrons into the conduction bands. The most significant difference between the $\mathrm{Si}$ and GaAs simulations is that in the case of GaAs the laser pulse acts to transfer charge from the As anions to Ga cations. The charge transfer along with a relaxation of the surface atoms acts as a stabilizing mechanism for the GaAs surface, and no ablation is observed. To ablate the GaAs (110) surface, the laser fluence was increased to $2.1 \mathrm{~kJ} / \mathrm{m}^{2}$. Due to the highly nonlinear interaction between the photons and the crystal, 13.8 percent of the electrons were excited into the conduction bands. This level of excitation was far greater than the minimum needed for ablation. Unfortunately, we did not have time to search out and simulate fluences closer to the minimum. The result was an extreme and rapid disruption of the lattice, as shown in figure 5. The snapshot was taken $1 \mathrm{ps}$ into the simulation. The ablated material contains mostly monomers, however dimmers, trimmers, 4-atom and 6-atom units are also observed, as shown more clearly in the slightly tilted view of Fig. 6. 


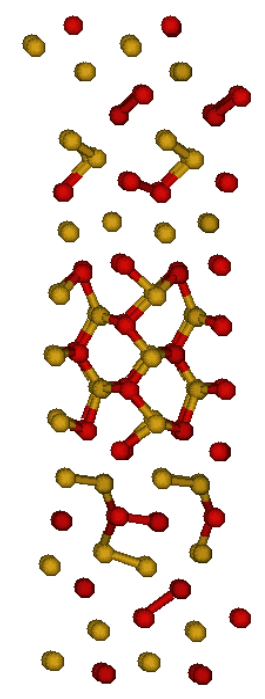

FIG. 5: The GaAs supercell, as viewed from the (110) direction, 1 ps after the onset of the laser pulse.

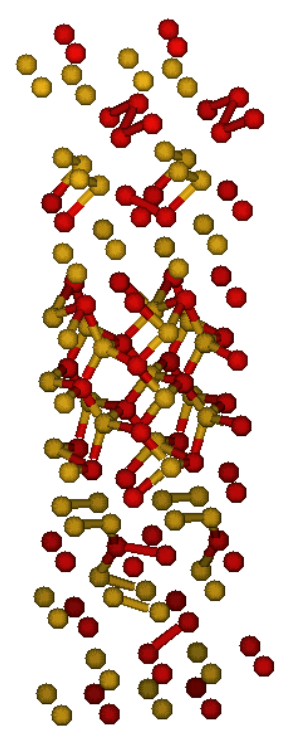

FIG. 6: Same as figure 5 except slightly tilted so as to reveal the structure of the ablated material.

Along with the information obtained from viewing the actual atomic motions in the animations, we also have access to data on the kinetic energy, pair correlation function, 
and all other information normally obtainable form classical molecular dynamics simulations. The above simulations clearly show the promise of obtaining detailed microscopic information on the laser ablation process.

\section{Conclusion:}

Our experimental results suggest that the stoichiometries of the PLD multielement nanoclusters are closer with those of the targets when lasers having pulse lengths shorter than 25 ps are used. However, this does not imply that simply ablating a multielement target in vacuum with pulses shorter than 25 ps will automatically result in the formation of stoichiometric nanocrystals. The preservation of the stoichiometry of the irradiated target and the formation of stoichiometric semiconductor nanocrystals require ablating the targets with short laser pulses in a background gas, with the minimum pressure being material dependent. The background gas serves to cool down the ablated target following the intense laser irradiation and to scatter away the atomic species and small sub-stoichiometric nanoclusters from the collected nanocrystal films. For metal alloys, the stoichiometry of the ablated target cannot be found in the collected nanocluster films if the constituent elements have widely different vapor pressures. On the computational front, we have successfully used density-functional based tight-binding potentials to study the femtosecond laser/GaAs target interaction. This knowledge is useful in helping us to explain some of our experimental results as well as to plan further experiments. In addition, the code work developed here can also be extended to other multi-element compounds/alloys as well.

\section{Acknowledgement:}

This work was performed under the auspices of the U.S. Department of Energy by the University of California, Lawrence Livermore National Laboratory, under contract No. W-7405-ENG-48.

\section{References:}

1. L. Banyai, S. W. Koch, Semiconductor Quantum dots (World Scientific, Singapore, 1993).

2. A. P. Alivisatos, Semiconductor clusters, nanocrystals, and quantum dots, science 271, 933 (1996).

3. T. VanBuuren, L. N. Dinh, L. L. Chase, W. J. Siekhaus, L. J. Terminello, Changes in the electronic properties of Si nanocrystals as a function of particle size, Phys. Rev. Lett. 80, 3803 (1998).

4. L. N. Dinh, S. E. Hayes, A. E. Wynne, M. A. Wall, C. K. Saw, B. C. Stuart, M. Balooch, A. K. Paravatsu, J. A. Reimer, Properties of GaAS nanoclusters deposited by a femtosecond laser, J. Mater. Scie. 37, 3953 (2002).

5. T. W. Trelenberg, L. N. Dinh, C. K. Saw, B. C. Stuart, M. Balooch, Femtosecond pulsed laser ablation of GaAs, submitted to Phys. Rev. B.

6. T. W. Trelenberg, L. N. Dinh, B. C. Stuart, M. Balooch, Femtosecond pulsed laser ablation of metal alloy and compound-semiconductor targets, to be submitted to J. Appl. Phys.

7. B. Torralva, T. A. Niehaus, M. Elstner, S. Suhai, Th. Frauenheim, R. E. Allen, Response of $C_{60}$ and $C_{n}$ to ultrashort laser pulses, Phys. Rev. B 64, 153105 (2001).

8. B. Torralva and P. E. A. Turchi, unpublished. 